\title{
Symposium 10: Correlates of visual motion processing: ERP and brain imaging Organized by M. Niedeggen
}

\section{Symp 10/1}

\section{SELECTIVE DISORDERS OF DIFFERENT KINDS OF VISUAL MOTION PERCEPTION}

\author{
A. Cowey ${ }^{\prime}$ and L.M. Vaina ${ }^{2}$ \\ ${ }^{\prime}$ University of Oxford, Department of Experimental Psychology, South Parks Road, Oxford OXI 3UD, \\ $U K$ and ${ }^{2}$ Boston University, Department of Biomedical Engineering, 44 Cummington Street, Boston, MA \\ 02215, USA
}

Several functional imaging experiments indicate that multiple cortical visual areas are activated when subjects are carrying out perceptual tasks involving the detection and discrimination of motion. Yet the perceptual awareness of motion per se and its discrimination is almost abolished by a large cortical lesion that involves human visual area MT/V5, but does not involve some of the regions activated by moving stimuli in normal observers. We have therefore carried out a series of studies of the role of different regions in different forms of motion perception. Patient FD, who has a small unilateral cortical lesion slightly dorsal to area MT/V5 has normal thresholds in his impaired contralateral field for the detection of global first-order (Fourier) motion but is markedly impaired when second-order (non-Fourier) versions of otherwise identical displays are used. In contrast, patient RA who has a unilateral lesion centred on what are probably extrastriate visual areas V2 and V3 in the dorso-medial part of the occipital lobe, has the complementary dissociation of symptoms in his impaired hemifield. He is markedly impaired at discriminating first-order but not second-order motion. The results indicate that at some stage in motion perception, separate cortical regions underlie the processing of first and second-order motion. A third patient, $\mathrm{AL}$, perceives the motion per se in moving displays but is unable to use it to create form from motion, even though she can perceive and discriminate the stationary forms when they are defined by, for example, luminance contrast. She is also unable to perceive the Johansson illusion, whereas the wellknown motion blind patient LM, whose lesion involves MT/V5 does perceive the Johansson illusion. The lesion in patient $\mathrm{AL}$ involves the fusiform and lingual gyri and other regions of the ventral aspect of the temporal lobe but spares area MT/V5. The pattern of results in patients FD, RA and AL is consistent with the neuroimaging studies that reveal multiple cortical areas involved in the processing of motion. They are also consistent with results of single cell recording in awake behaving monkeys, where regional functional specialisation is established. For example, biological motion is well represented by cells in parts of the rostral superior temporal sulcus. However, attempts by means of functional neuroimaging to demonstrate regional specialisation with respect to different forms of motion processing have not yet revealed such clear differences. The reasons for the discrepancy between the results from functional neuroimaging and those from single cell recordings and neurological patients remain unclear. 


\section{Symp 10/2 \\ FMRI STUDIES OF MOTION PROCESSING IN HUMAN OCCIPITAL CORTEX}

A.T. Smith ${ }^{l}$, K.D. Sing $h^{l}$, and M.W. Greenle ${ }^{2}$

${ }^{I}$ Department of Psychology, Royal Holloway, University of London, UK and ${ }^{2}$ Neurologische Klinik, University of Freiburg, Germany

Moving visual stimuli have been used in numerous $\mathrm{AMRI}$ studies of the human cerebral cortex. They invariably activate large swathes of occipital cortex, including all the well-defined retinotopic visual areas. They also commonly activate a variety of other brain areas, including such disparate regions as the posterior parietal cortex, anterior cingulate cortex and the cerebellum. But we must assume that much of this activity is not truly a response to motion. Moving images always contain spatial structure and a significant portion of the neural response can presumably be attributed to the spatial, rather than temporal properties of the stimulus. Some fMRI studies have attempted to quantify motion specificity by comparing responses to moving and stationary versions of the same stimulus. But this does not seem appropriate. Neurophysiological studies of primate cortex show us that many neurons respond well to temporal modulation but are not very sensitive to direction. So part of the fMRI response may arise from temporal luminance modulation at point locaticns in the image, rather than from movement per se, leading to an over-estimation of motion sensitivity when moving and stationary responses are compared. We have investigated the activation produced in occipital cortex in response to presentation of spatially extensive drifting and counterphasing gratings of various spatial and temporal frequencies. We also used anatomical MRI images to simulate the flattening of occipital cortex and standard retinotopic mapping procedures to identify the boundaries between the various visual areas (V1, V2v/d, V3/VP, V3A, V4). The grating responses were then projected onto the two-dimensional (flattened) cortical surface and averaged across the voxels within each visual region. We also examined V5, identified on single brain slices. Results varied little among the various visual areas. Temporal tuning functions were comparable to those obtained psychophysically, but spatial frequency functions were not, favouring lower spatial frequencies than might be expected. Comparisons of the activity produced by a drifting grating and a counterphase grating of the same spatial and temporal frequency yielded surprisingly low ratios. Such ratios seldom exceeded 1.5 , even in V5, and in many cases were not significantly different from 1.0 . If we assume that direction-sensitive neurons exist to detect motion and are unresponsive to counterphase flicker, then our results suggest that directional responses form a rather small part of the total response to temporal structure. However, if direction-specific neurons respond to one component of a counterphase grating and are ignore the other, then counterphase gratings will actually stimulate more directionsensitive neurons than will a drifting grating. Physiological data suggest that the truth is between the two. Quantifying motion sensitivity with fMRI is not straightforward and rests on the assumptions one chooses to make. 


\title{
Symp 10/3
}

\section{ERPS AND THE MECHANISMS OF MOTION ADAPTATION}

M. Bach*, D. Ullrich, T. Dorn, A. Unsöld, and M. Hoffmann

Univ.-Augenklinik, Freiburg, Germany

A number of recent studies have explored the mechanisms of human motion perception through the use of event related responses (ERPs), namely the visual evoked potential (VEP) elicited by motion onset. A number of interesting properties emerged, after discrepancies between various groups were resolved by recognizing that (1) the motion VEP is strongly affected by adaptation (can even change its polarity), and (2) the test stimulus itself drives the system into an adapted state, unless very low motion duty-cycles are employed (Bach \& Ullrich 1994). High contrast sensitivity (Müller \& Göpfert 1988, Bach \& Ullrich 1997) and a lateral localization of the source of the potentials (Probst et al. 1993) further support the hypothesis that a negativity around $160 \mathrm{~ms}$ after motion onset is the VEP component that reflects motion processing. We have examined the dynamic properties of motion adaptation and its direction-specificity: (1) Measurements of the time course of adaptation and recovery appear reasonably fitted by a single time constant which is about $3 \mathrm{~s}$ for adaptation and 3x longer for recovery (Hoffmann et al. 1999). These results were similar using both psychophysical and electrophysiological techniques (2) We assessed the direction-specificity of motion adaptation in the motion-VEP over 8 directions "around the clock" and found two effects that reduced the VEP amplitude: (a) a non-direction specific, global adaptation effect which reduces the motion-VEP by $50 \%$, and (b) a specific effect, which reduced the amplitude in the adapting direction by a further $25 \%$ (Unsöld et al. 1998). A global effect also resulted after pattern-onset stimulation. This strengthens the interpretation that the global adaptation effect does not result from motion-specific mechanisms.

(3) We extended the adaptation paradigm to the human retina, simultaneously recording retinal (ERG) and cortical potentials (VEP). In the retinal potential we also found a strong global effect (of $30 \%$ amplitude reduction), but no significant direction-specific effect. Thus no specific motion processing seems to occur in the human retina. In conclusion, motion-evoked potentials in the human electroencephalogram have proven to be a useful tool: They bridge the gap between single-cell studies in animals and human psychophysics with a high temporal resolution and can produce insights unavailable elsewhere. With this tool we are currently assessing the effect of attention on motion processing.

Support of the Deutsche Forschungsgemeinschaft is gratefully acknowledged

\section{Symp 10/4}

\section{ERP CORRELATES OF MOTION COHERENCE AND TRANSPARENCY}

\author{
M. Niedeggen \\ Institute of Physiological Psychology II, Heinrich-Heine-University, D-40225 Duesseldorf, Germany
}

Psychophysical studies and single unit recordings have shown that the perception of coherent visual motion requires the analysis of the incoming motion signals followed by an integration of these local 

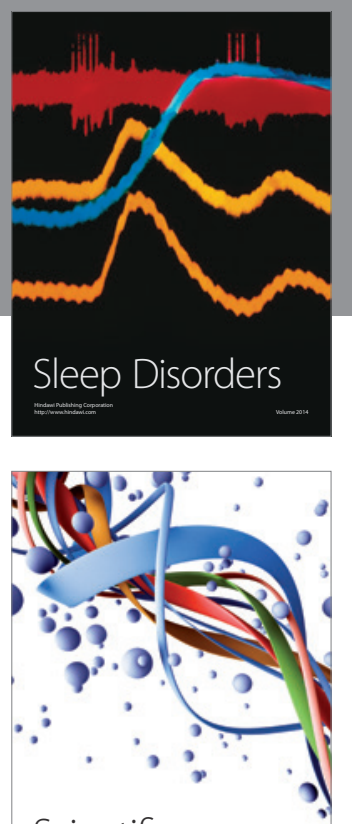

Scientifica
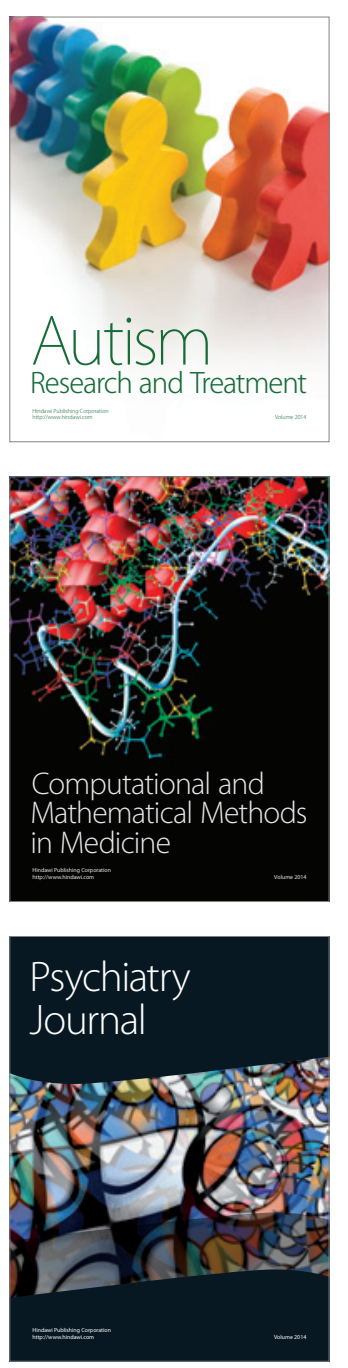
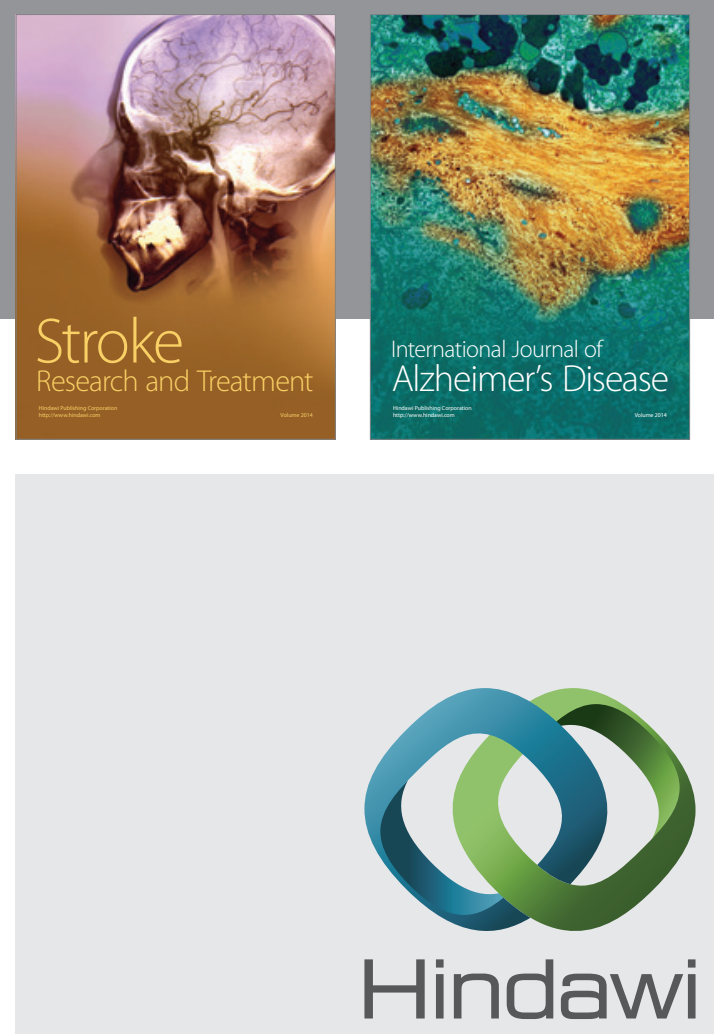

Submit your manuscripts at

http://www.hindawi.com
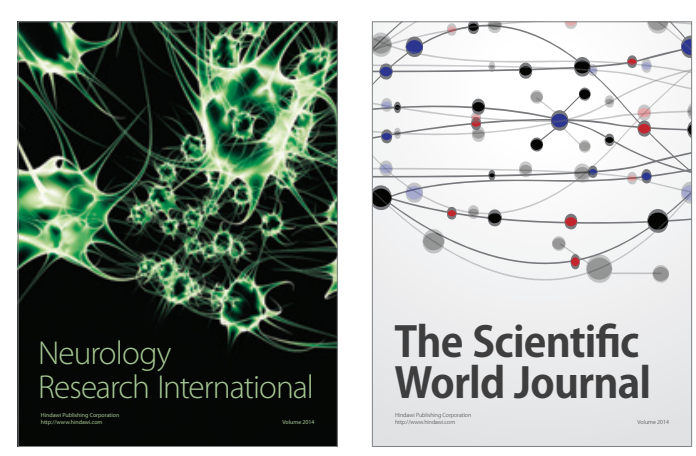

The Scientific World Journal

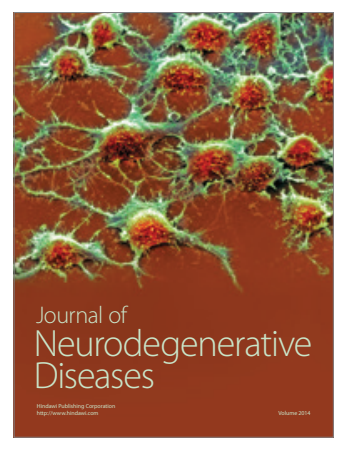

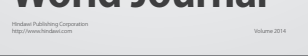

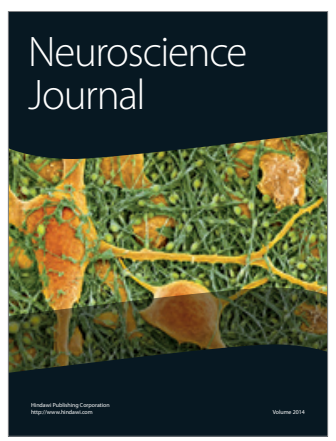

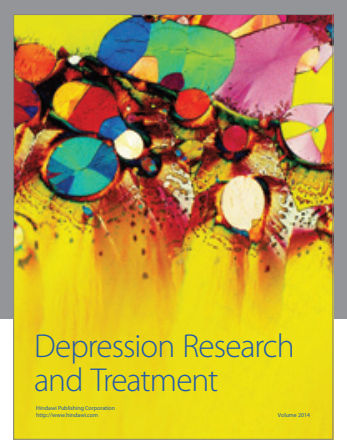
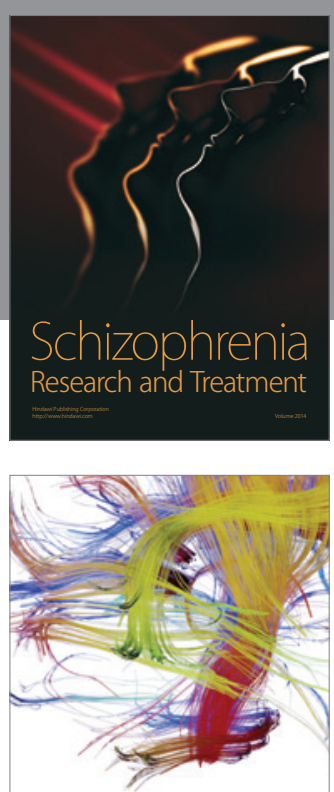

Brain Science

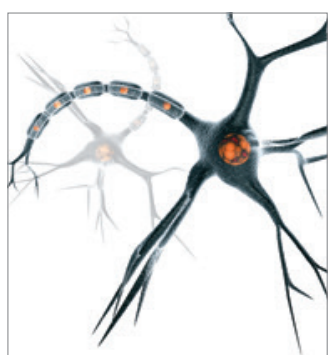

Neural Plasticity
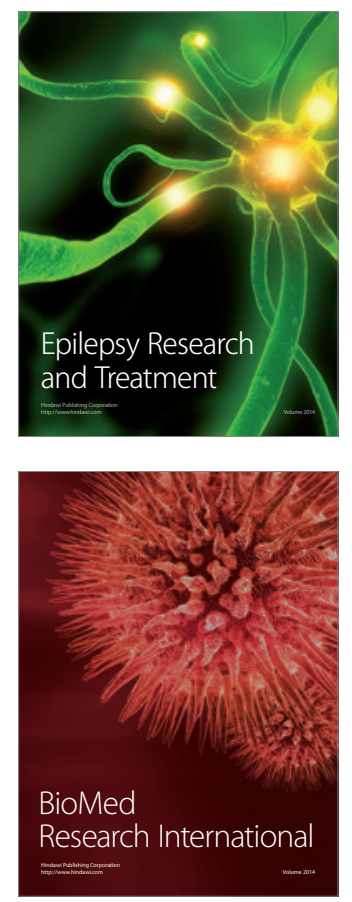

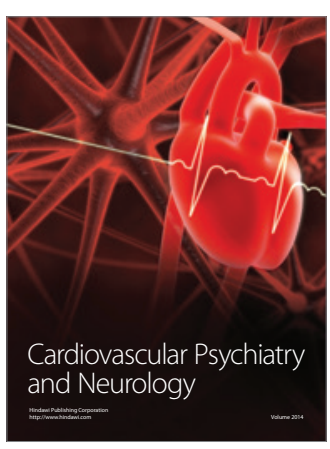

Parkinson's

Disease
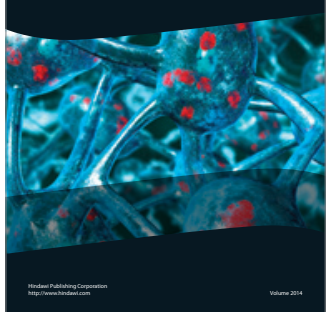